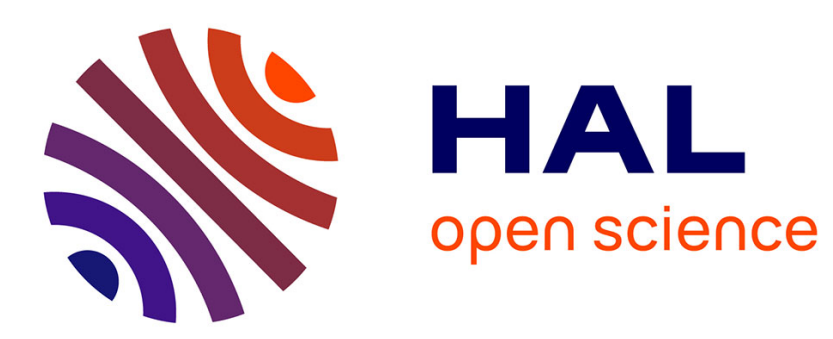

\title{
The implicit discretization of the super-twisting sliding-mode control algorithm
}

Bernard Brogliato, Andrey Polyakov, Denis Efimov

\section{To cite this version:}

Bernard Brogliato, Andrey Polyakov, Denis Efimov. The implicit discretization of the super-twisting sliding-mode control algorithm. IEEE Transactions on Automatic Control, 2020, 65 (8), pp.3707-3713. 10.1109/TAC.2019.2953091 . hal-02336599

\section{HAL Id: hal-02336599 \\ https://hal.inria.fr/hal-02336599}

Submitted on 29 Oct 2019

HAL is a multi-disciplinary open access archive for the deposit and dissemination of scientific research documents, whether they are published or not. The documents may come from teaching and research institutions in France or abroad, or from public or private research centers.
L'archive ouverte pluridisciplinaire HAL, est destinée au dépôt et à la diffusion de documents scientifiques de niveau recherche, publiés ou non, émanant des établissements d'enseignement et de recherche français ou étrangers, des laboratoires publics ou privés. 


\title{
The implicit discretization of the super-twisting sliding-mode control algorithm
}

\author{
Bernard Brogliato $^{1}$, Andrey Polyakov ${ }^{2}$ and Denis Efimov ${ }^{2}$
}

\begin{abstract}
This paper deals with the analysis of the time-discretization of the super-twisting algorithm, with an implicit Euler method. It is shown that the discretized system is well-posed. The existence of a Lyapunov function with convex level sets is proved for the continuoustime closed-loop system. Then the global asymptotic Lyapunov stability of the unperturbed discrete-time closedloop system is proved. The convergence to the origin in a finite number of steps is proved also in the unperturbed case. Numerical simulations demonstrate the superiority of the implicit method with respect to an explicit discretization with significant chattering reduction.
\end{abstract}

Keywords: Implicit Euler discretization; sliding mode control; super-twisting algorithm; Lyapunov stability.

\section{INTRODUCTION}

The sliding mode algorithms found their place in the theory and practice of control and estimation [10]. Among them, the super-twisting algorithm is one of the most popular higher-order sliding mode (HOSM) tools [20], [6]. The time discretization of set-valued sliding-mode control (SMC), has been recognized as being a major issue as it leads to input and ouput chattering (so-called digital or numerical chattering) in the case of an explicit discretization [11], [7]. Another method has been introduced in [1], [2], [12], see also [15], [3] for a similar approach. It is based on an implicit discretization of the continuous-time system. It has been shown theoretically and experimentally to provide significant chattering alleviation [25], [13], [12], [14], without modifying the controller's structure. The implicit discretization applies to various systems and controllers: first-order SMC for linear

A preliminary version of this article was presented at the 15th Int. Workshop on Variable Structure Systems VSS'2018, Graz, Austria, July 2018. This work was supported by the ANR project Digitslid ANR-18-CE40-0008-01.

${ }^{1}$ Bernard Brogliato is with Univ. Grenoble Alpes, Inria, CNRS, Grenoble INP, LJK, 38000 Grenoble, France. bernard.brogliatodinria.fr

${ }^{2}$ Andrey Polyakov and Denis Efimov are with Inria, Univ. Lille, CNRS, UMR 9189 - CRIStAL, F-59000 Lille, France and Department of Control Systems and Informatics, University ITMO, 49 av. Kronverkskiy, 197101 Saint Petersburg, Russia. andrey.polyakov (denis.efimov) Cinria.fr time-invariant systems with matching disturbances [1], [2], [12] or parameter uncertainties [19], nonlinear Lagrangian systems with matching disturbances and parameter uncertainties [18], twisting algorithm [2], [13]. Another very interesting feature of the implicit discretization is that it yields global Lyapunov finitetime stability [12], [4], [8], contrarily to the explicit method which guarantees local stability only [8], [17] which may be quite problematic in applications, in addition to digital chattering.

It is noteworthy that all the above cited articles, show that the so-called emulation of a feedback controller yields poor results if an explicit Euler method is used to discretize SMC, whereas most of the performances and stability properties are preserved if the controller is emulated with an implicit method. In addition the input converges to its set-valued continuous-time counterpart [12, Propositions 3 and 4].

The aim of this note is to present the analysis of the implicit discretization of the super-twisting algorithm using the implicit Lyapunov function approach. It is organised as follows: section II introduces the supertwisting scheme, and shows the existence of a strict Lyapunov function with convex level sets; section III proposes an implicit discretization of the continuoustime scheme, and shows its well-posedness (nonanticipativity, existence and uniqueness of the controller); section IV is dedicated to the stability analysis of the discrete-time closed-loop system; the results of simulation are presented in section $\mathrm{V}$; conclusions end the paper in section VI.

a) Notations and definitions: The set-valued sign function is defined as $\operatorname{sgn}(x)=1$ if $x>0,-1$ if $x<0$, and $[-1,1]$ if $x=0$. Let $K \subseteq \mathbb{R}^{n}$ be a closed non empty convex set. Its normal cone at $x \in K$ is defined as $\mathcal{N}_{K}(x)=\left\{w \in \mathbb{R}^{n} \mid\langle w, y-x\rangle \leq 0\right.$ for all $\left.y \in K\right\}$. In particular $\mathcal{N}_{[-1,1]}(s)=\left\{\begin{array}{lll}\mathbb{R}^{-} & \text {if } & s=-1 \\ \{0\} & \text { if } & s \in(-1,1) . \\ \mathbb{R}^{+} & \text {if } & s=1\end{array}\right.$. It follows from Convex Analysis that $\mathcal{N}_{[-1,1]}(s)$ is the inverse of the sign set-valued function, that is: $x \in$ $\operatorname{sgn}(y) \Leftrightarrow y \in \mathcal{N}_{[-1,1]}(x)$, for all reals $x$ and $y$. Let $G: \mathbb{R}^{n} \rightrightarrows \mathbb{R}^{m}$ be a set-valued mapping, and $f$ : $\mathbb{R}^{n} \rightarrow \mathbb{R}^{m}$ be a single-valued mapping. Then $0 \in$ 
$f(x)+G(x)$ is a generalized equation.

\section{THE SUPER-TWISTING ALGORITHM}

\section{A. The control algorithm}

The closed-loop system we are dealing with is given as [16], [20], [23]:

$$
\left\{\begin{array}{l}
\dot{x}_{1}(t)=-\lambda_{1} \sqrt{\left|x_{1}(t)\right|} \operatorname{sgn}\left(x_{1}(t)\right)+x_{2}(t) \\
\dot{x}_{2}(t) \in-\lambda_{2} \operatorname{sgn}\left(x_{1}(t)\right)+\Delta(t) .
\end{array}\right.
$$

It stems from the application of the super-twisting controller given by:

$$
\left\{\begin{array}{l}
u(t)=-\lambda_{1} \sqrt{\left|x_{1}(t)\right|} \operatorname{sgn}\left(x_{1}(t)\right)+\nu(t), \\
\dot{\nu}(t) \in-\lambda_{2} \operatorname{sgn}\left(x_{1}(t)\right)
\end{array}\right.
$$

applied to the plant

$$
\dot{x}_{1}(t)=u+\varphi(t), \quad \dot{\varphi}(t)=\Delta(t)
$$

with the definition of the (unmeasurable) state $x_{2} \triangleq$ $\nu+\varphi$, and $\sup _{t \geq 0}|\Delta(t)| \leq L$ for some known constant $L$. Notice that in the undisturbed case $\varphi(t) \equiv 0$ one has $x_{2}(t)=\nu(t)$ for all $t \geq 0$.

\section{B. Global asymptotic Lyapunov stability}

The finite-time stability of the closed-loop system (1) has been analysed in [20], [23], [24], where different Lyapunov functions have been exhibited. A crucial property to transport the Lyapunov stability properties from the continuous-time system to the discretized system, is that the (continuous-time) Lyapunov function has convex level sets. It is not obvious that the Lyapunov functions proposed in [20], [23], [24] satisfy such a property (though some numerical calculations suggest that this could be the case). In addition, our further constructions also require a smoothness of Lyapunov functions. This is not the case for Lyapunov functions obtained in [20], [23], [24].

Lemma 1. If $L \geq 0, \lambda_{1}>\sqrt{4 \sqrt{2} L}$ and $L<$ $\lambda_{2}<\frac{\lambda_{1}^{2}}{2 \sqrt{2}}-L$, then the system (1) admits $a$ strict Lyapunov function $V \in C\left(\mathbb{R}^{n},[0,+\infty) \cap\right.$ $C^{1}\left(\mathbb{R}^{n} \backslash\{0\},(0,+\infty)\right)$, with ellipsoidal level sets.

Proof. I. Let us study initially the case $0 \leq L<\frac{1}{2}$, $L<\lambda_{2}<1-L$ and $\lambda_{1}=\sqrt{2 \sqrt{2}}$. Let us consider the implicit Lyapunov function (ILF) candidate $V(\cdot)$ for the super-twisting system given by the equality $Q(V, x)=0$ where (see e.g., [22] for details)

$$
Q(V, x)=x^{\top} D\left(V^{-1}\right) P D\left(V^{-1}\right) x-1,
$$

where $x=\left(x_{1}, x_{2}\right)^{\top} \in \mathbb{R}^{2}, D\left(V^{-1}\right)=\left(\begin{array}{cc}V^{-2} & 0 \\ 0 & V^{-1}\end{array}\right)$, $P=\left(\begin{array}{cc}1+\varepsilon & -1 \\ -1 & 1\end{array}\right)$ is a positive definite matrix for any $\varepsilon>0$. The considered Lyapunov candidate is welldefined and positive definite if the following LMI holds [22]

$$
P G_{D}+G_{D}^{\top} P \succ 0
$$

where $G_{D}=\left(\begin{array}{ll}2 & 0 \\ 0 & 1\end{array}\right)$. The latter matrix inequality holds if $\varepsilon>\frac{1}{8}$. The partial derivative of $V(\cdot)$ is given by

$$
\frac{\partial V}{\partial x}=\frac{2 V x^{\top} D\left(V^{-1}\right) P D\left(V^{-1}\right)}{x^{\top} D\left(V^{-1}\right)\left(P G_{D}+G_{D}^{\top} P\right) D\left(V^{-1}\right) x},
$$

Let us denote

$$
F(x)=\left(\begin{array}{c}
-\lambda_{1} \sqrt{\left|x_{1}\right|} \operatorname{sgn}\left(x_{1}\right)+x_{2} \\
-\lambda_{2} \operatorname{sgn}\left(x_{1}\right)+[-L, L]
\end{array}\right),
$$

where $\lambda_{1}, \lambda_{2}$ and $L$ are defined in (1). Note that the autonomous system $\dot{x} \in F(x)$ includes the timevarying system (1) as particular case since $|\Delta(t)| \leq L$ [21]. Let us estimate the time derivative of the ILF candidate along the trajectories of the system (1)

$$
\begin{gathered}
\sup _{\xi \in F(x)} \frac{\partial V}{\partial x} \xi=\frac{2 V \sup _{\xi \in F(x)} x^{\top} D\left(V^{-1}\right) P D\left(V^{-1}\right) \xi}{x^{\top} D\left(V^{-1}\right)\left(P G_{D}+G_{D}^{\top} P\right) D\left(V^{-1}\right) x} \\
=\frac{2 \sup _{z \in F\left(D\left(V^{-1}\right) x\right)} x^{\top} D\left(V^{-1}\right) P z}{x^{\top} D\left(V^{-1}\right)\left(P G_{D}+G_{D}^{\top} P\right) D\left(V^{-1}\right) x},
\end{gathered}
$$

where $x^{\top} D\left(V^{-1}\right) P D\left(V^{-1}\right) x=1$. Obviously, due to condition (4), the latter fraction is negative if

$$
\sup _{z \in F(y)} y^{\top} P z<0 \quad \text { for } \quad y^{\top} P y=1 \text {. }
$$

If $y_{1}=0$, then $y^{\top} P y=1$ implies $y_{2}^{2}=1$ and $F(y)=$ $\left(\left[-\lambda_{2}, \lambda_{2}\right]+[-L, L]\right)$. Hence, $\sup _{z \in F(y)} y^{\top} P z=-1+$ $\left(\lambda_{2}+L\right)<0$.

Let us consider now the case $y_{1}>0$ (the case $y_{1}<0$ can be treated similarly). One has

$$
\begin{gathered}
\sup _{z \in F(y)} y^{\top} P z=\sup _{\tilde{L} \in[-L, L]} y^{\top} P\left(\begin{array}{c}
-\lambda_{1} \sqrt{y_{1}}+y_{2} \\
-\lambda_{2}+\tilde{L}
\end{array}\right)= \\
\sup _{\tilde{L} \in[-L, L]}-\lambda_{1}(1+\varepsilon) y_{1}^{\frac{3}{2}}+\lambda_{1} y_{2} \sqrt{y_{1}}+(1+\varepsilon) y_{1} y_{2}-y_{2}^{2} \\
+\left(y_{1}-y_{2}\right)\left(\lambda_{2}-\tilde{L}\right) .
\end{gathered}
$$

The identity $y^{\top} P y=1$ gives $y_{2}^{2}-2 y_{1} y_{2}+(1+\varepsilon) y_{1}^{2}=$ 1 and

$$
\begin{array}{ll}
y_{2}=y_{1}-\sqrt{1-\varepsilon y_{1}^{2}} \quad \text { if } \quad & y_{1}-y_{2} \geq 0, \\
y_{2}=y_{1}+\sqrt{1-\varepsilon y_{1}^{2}} \quad \text { if } & y_{1}-y_{2}<0,
\end{array}
$$

with $y_{1} \in\left(0, \frac{1}{\sqrt{\varepsilon}}\right]$. Hence, for $y_{1}-y_{2} \geq 0$ we derive

$$
\sup _{z \in F(y)} y^{\top} P z \leq q_{1}\left(y_{1}\right)-\left(1-\lambda_{2}-L\right) \sqrt{1-\varepsilon y_{1}^{2}}
$$


where

$$
\begin{gathered}
q_{1}\left(y_{1}\right)=-\lambda_{1} \varepsilon y_{1}^{\frac{3}{2}}-\lambda_{1} \sqrt{y_{1}} \sqrt{1-\varepsilon y_{1}^{2}}+2 \varepsilon y_{1}^{2} \\
\quad+(1-\varepsilon) y_{1} \sqrt{1-\varepsilon y_{1}^{2}}+\sqrt{1-\varepsilon y_{1}^{2}}-1 .
\end{gathered}
$$

For $y_{1}-y_{2}<0$ we have

$$
\sup _{z \in F(y)} y^{\top} P z \leq q_{2}\left(y_{1}\right)-\left(\lambda_{2}-L\right) \sqrt{1-\varepsilon y_{1}^{2}}
$$

where

$$
\begin{gathered}
q_{2}\left(y_{1}\right)=-\lambda_{1} \varepsilon y_{1}^{\frac{3}{2}}+\lambda_{1} \sqrt{y_{1}} \sqrt{1-\varepsilon y_{1}^{2}}+2 \varepsilon y_{1}^{2}-1 \\
+(\varepsilon-1) y_{1} \sqrt{1-\varepsilon y_{1}^{2}} .
\end{gathered}
$$

If $\varepsilon=1$ and $\lambda_{1}=\sqrt{2 \sqrt{2}}$ then

$$
q_{1}(0)=0, \quad q_{1}\left(y_{1}\right)<0 \text { for } y_{1} \in(0,1],
$$

$q_{2}\left(\frac{1}{\sqrt{2}}\right)=0, \quad q_{2}\left(y_{1}\right)<0$ for $y_{1} \in\left(0, \frac{1}{\sqrt{2}}\right) \cup\left(\frac{1}{\sqrt{2}}, 1\right]$.

The detailed proof of the latter inequalities is omitted for shortness. The graphs of the functions over the interval $[0,1]$ are depicted in Fig. 1. Therefore, $Q$ with

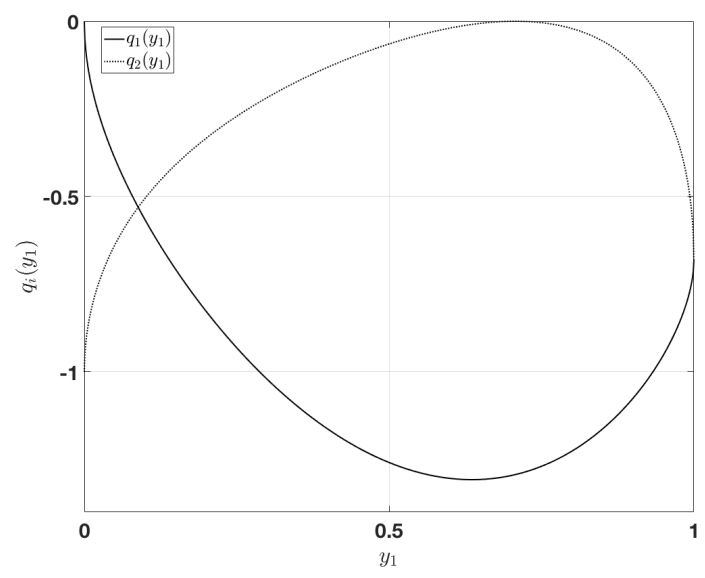

Fig. 1. Graphs of $q_{1}(\cdot)$ (solid line) and $q_{2}\left({ }^{\cdot}\right)$ (dashed line) for $\varepsilon=1, \lambda_{1}=\sqrt{2 \sqrt{2}}$.

$P=\left(\begin{array}{cc}2 & -1 \\ -1 & 1\end{array}\right)$ is the implicit Lyapunov function for (1) with $\lambda_{1}=\sqrt{2 \sqrt{2}}$ and $L<\lambda_{2}<1-L$, i.e., there exists a positive definite function $V: \mathbb{R}^{n} \rightarrow[0,+\infty)$ that is strict Lyapunov function to (1) (the notion of strict Lyapunov function is given in Appendix) and $Q(V(x), x)=0$ for all $x \in \mathbb{R}^{n}$. The level sets of $V(\cdot), \Omega(r)=\left\{x \in \mathbb{R}^{2} \mid V(x) \leq r\right\}$, are given by (see e.g., [22])

$$
\Omega(r)=\left\{x \in \mathbb{R}^{2} \mid x^{\top} D\left(r^{-1}\right) P D\left(r^{-1}\right) x \leq 1\right\} .
$$

Obviously, $\Omega(r)$ is an ellipsoid for any $r>0$.

II. Now, let $\lambda_{1}, \lambda_{2}$ and $L$ satisfy the conditions of the lemma. The linear change of coordinates $x^{\text {new }}=$ $\frac{2 \sqrt{2}}{\lambda_{1}^{2}} x$, transforms (1) to

$$
\begin{aligned}
& \dot{x}_{1}^{\text {new }}=-\sqrt{2 \sqrt{2}} \sqrt{\left|x_{1}^{\text {new }}\right|} \operatorname{sgn}\left(x_{1}^{\text {new }}\right)+x_{2}^{\text {new }} \\
& \dot{x}_{2}^{\text {new }}=-\frac{2 \sqrt{2} \lambda_{2}}{\lambda_{1}^{2}} \operatorname{sgn}\left(x_{1}^{\text {new }}\right)+\frac{2 \sqrt{2} \Delta}{\lambda_{1}^{2}},
\end{aligned}
$$

where $\frac{2 \sqrt{2}|\Delta(t)|}{\lambda_{1}^{2}} \leq \tilde{L}:=\frac{2 \sqrt{2} L}{\lambda_{1}^{2}}<\frac{1}{2}$, and $\tilde{L}<\frac{2 \sqrt{2} \lambda_{2}}{\lambda_{1}^{2}}<1-$ $\tilde{L}$ by assumptions of the lemma. As it has been proven above, the transformed system has a strict Lyapunov function $V^{\text {new }}: \mathbb{R}^{n} \rightarrow[0,+\infty)$ with ellipsoidal level sets. Then, $V(x)=V^{\text {new }}\left(\frac{2 \sqrt{2}}{\lambda_{1}^{2}} x\right), x=\left(x_{1}, x_{2}\right)$ defines the strict Lyapunov function for the original system. Finally, notice that the linear transformation of coordinates keeps the level sets of the Lyapunov function ellipsoidal.

For any $L>0$, we can always select parameters $\lambda_{1}$ and $\lambda_{2}$ satisfying Lemma 1 . In other words, the supertwisting system always admits a smooth Lyapunov function with ellipsoidal level sets, under some proper selection of parameters. The restrictions on these parameters are more conservative compared to [20], due to the requirements of convexity and smoothness of the corresponding Lyapunov function.

\section{THE SUPER-TWISTING ALGORITHM IMPLICIT DISCRETIZATION}

The controller is discretized as follows:

$$
\left\{\begin{array}{l}
u_{k}=-\lambda_{1} \sqrt{\left|\tilde{x}_{1, k+1}\right|} \operatorname{sgn}\left(\tilde{x}_{1, k+1}\right)+\nu_{k+1} \\
\nu_{k+1} \in \nu_{k}-\lambda_{2} h \operatorname{sgn}\left(\tilde{x}_{1, k+1}\right)
\end{array}\right.
$$

where $t_{k+1}-t_{k}=h>0$ is the time-step (or sampling period), and we denote $f\left(t_{k}\right)=f_{k}$. The variable $\tilde{x}_{1, k+1}$ is an intermediate variable, used to calculate the input, because $x_{1, k+1}$ is not available due to the unknown disturbance. It will be defined precisely later. The input in (5) is the control to be applied at $t=t_{k}$ and on the whole time interval $\left[t_{k}, t_{k+1}\right)$. The plant discretized model is chosen as:

$$
\left\{\begin{array}{l}
x_{1, k+1}=x_{1, k}+h u_{k}+h \bar{\varphi}_{k} \\
\varphi_{k+1}=\varphi_{k}+h \bar{\Delta}_{k}
\end{array}\right.
$$

where $\bar{\varphi}_{k}$ and $\bar{\Delta}_{k}$ are the discrete-time counterparts of $\varphi(t)$ and $\Delta(t)$ in (3), which depend on the used method. For instance, the simple explicit Euler method yields $\bar{\varphi}_{k}=\varphi_{k}=\varphi\left(t_{k}\right)$ and $\bar{\Delta}_{k}=\Delta_{k}=\Delta\left(t_{k}\right)$, while the exact discretization (zero-order hold) yields from the mean value theorem, assuming that $\Delta(\cdot)$ is continuous: $\bar{\varphi}_{k}=\varphi_{k}+\left(\tau-t_{k}\right) \Delta(\gamma)$ and $\bar{\Delta}_{k}=\Delta(s)$, for some $s, \tau \in\left(t_{k}, t_{k+1}\right), \gamma \in\left(t_{k}, \tau\right)$. This may be extended to piecewise continuous perturbations. Notice 
that when $\Delta(t) \equiv 0$, then all methods yield the same discretization. Let us set:

$$
\tilde{x}_{1, k+1}=x_{1, k}+h u_{k},
$$

which plays the role of an unperturbed nominal plant, so that $\tilde{x}_{1, k}$ is a dummy variable which can be interpreted as the state of the unperturbed plant, and $\tilde{x}_{1, k+1}$ is available at $t=t_{k}$ as shown next. Inserting (5) into (7) we obtain:

$$
\left\{\begin{array}{l}
\tilde{x}_{1, k+1}=x_{1, k}-h \lambda_{1} \sqrt{\left|\tilde{x}_{1, k+1}\right|} \operatorname{sgn}\left(\tilde{x}_{1, k+1}\right)+h \nu_{k+1} \\
\nu_{k+1} \in \nu_{k}-\lambda_{2} h \operatorname{sgn}\left(\tilde{x}_{1, k+1}\right),
\end{array}\right.
$$

which is the unperturbed "virtual" closed-loop system. We rewrite (8) equivalently as:

$$
\begin{gathered}
\tilde{x}_{1, k+1} \in x_{1, k}-h \lambda_{1} \sqrt{\left|\tilde{x}_{1, k+1}\right|} \operatorname{sgn}\left(\tilde{x}_{1, k+1}\right) \\
+h \nu_{k}-h^{2} \lambda_{2} \operatorname{sgn}\left(\tilde{x}_{1, k+1}\right) .
\end{gathered}
$$

Let us recall that $\operatorname{sgn}(0)=[-1,1]$ so that indeed the right-hand side of (9) is set-valued, and (9) is a generalized equation with unknown $\tilde{x}_{1, k+1}$. Let us introduce the variable (a selection): $\xi_{k+1} \in \operatorname{sgn}\left(\tilde{x}_{1, k+1}\right)$. The controller $u_{k}$ to be calculated at $t=t_{k}$ and to be applied on $\left[t_{k}, t_{k+1}\right)$ is given as follows:

$$
\left\{\begin{array}{l}
u_{k}=-\lambda_{1} \sqrt{\left|\tilde{x}_{1, k+1}\right|} \xi_{k+1}+\nu_{k+1} \\
\nu_{k+1}=\nu_{k}-\lambda_{2} h \xi_{k+1},
\end{array}\right.
$$

Notice that (10) is just a rewriting of (5). Rewriting (9) we also obtain the generalized equation:

$$
g\left(\tilde{x}_{1, k+1}\right) \in-\lambda_{2} h^{2} \operatorname{sgn}\left(\tilde{x}_{1, k+1}\right),
$$

with $g(x) \triangleq x+a \sqrt{|x|} \operatorname{sgn}(x)+b_{k}, a \triangleq h \lambda_{1}>$ $0, \quad b_{k} \triangleq-x_{1, k}-h \nu_{k}$. The variable $\xi_{k+1}$ is the solution of the generalized equation

$$
f\left(-\lambda_{2} h^{2} \xi_{k+1}\right) \in \mathcal{N}_{[-1,1]}\left(\xi_{k+1}\right),
$$

where the function $f(\cdot)$ is defined as:

$$
f(y)=g^{-1}(y)= \begin{cases}\left(\frac{-a+\sqrt{a^{2}-4\left(b_{k}-y\right)}}{2}\right)^{2} & \text { if } y \geq b_{k} \\ -\left(\frac{a-\sqrt{a^{2}+4\left(b_{k}-y\right)}}{2}\right)^{2} & \text { if } y<b_{k} .\end{cases}
$$

Let us prove that the inclusion (12) follows from (11) by inversion of set-valued mappings: we have $g\left(\tilde{x}_{1, k+1}\right)=-\lambda_{2} h^{2} \xi_{k+1} \in-\lambda_{2} h^{2} \operatorname{sgn}\left(\tilde{x}_{1, k+1}\right)$. Now $\xi_{k+1} \in \operatorname{sgn}\left(\tilde{x}_{1, k+1}\right) \Leftrightarrow \tilde{x}_{1, k+1} \in \mathcal{N}_{[-1,1]}\left(\xi_{k+1}\right)$. Let $\beta_{k+1}$ be a selection of $\mathcal{N}_{[-1,1]}\left(\xi_{k+1}\right)$, then we can write $g\left(\beta_{k+1}\right)=-\lambda_{2} h^{2} \xi_{k+1}$, hence $\beta_{k+1}=$ $f\left(\begin{array}{lll}-\lambda_{2} & h^{2} & \xi_{k+1}\end{array}\right)$. Consequently $f\left(\begin{array}{lll}-\lambda_{2} & h^{2} & \xi_{k+1}\end{array}\right) \in$ $\mathcal{N}_{[-1,1]}\left(\xi_{k+1}\right)$ which is (12). The inversion of the function $g(\cdot)$ can be calculated from the fact that $g(x)=x+a \sqrt{x}+b_{k}$ if $x>0, g(x)=x-a \sqrt{-x}+b_{k}$ if $x<0$.

Remark 1. The controller $u_{k}$ in (10) is nonanticipative since both $\xi_{k+1}$ and $\tilde{x}_{1, k+1}$ are functions of known quantities at $t=t_{k}: h, \lambda_{1}, \lambda_{2}, \nu_{k}$ and $x_{1, k}$, both being solutions of the generalized equations (12) and (11).

The following holds:

Lemma 2. The generalized equations in (12) and (11) have a unique solution for any data $h, \lambda_{1}, \lambda_{2}, \nu_{k}$ and $x_{1, k}$.

Proof. Notice that both $f(\cdot)$ and $g(\cdot)$ are continuous. It readily follows from [9, Corollary 2.2.5] that (12) has at least one solution, and the set of solutions is compact. Let now $\zeta_{k+1} \triangleq-\lambda_{2} h^{2} \xi_{k+1}$, then (12) is equivalent to $0 \in f\left(\zeta_{k+1}\right)+\mathcal{N}_{\left[-\lambda_{2} h^{2}, \lambda_{2} h^{2}\right]}\left(\zeta_{k+1}\right)$. It can be checked that $\frac{d f}{d y}(y)>0$ for all $y \neq b$ while $\frac{d f}{d y}\left(b_{k}\right)=0$. Therefore applying [9, Proposition 2.3.2] and [9, Theorem 2.3.3], it follows that the generalized equation (12) always has a unique solution. This means that the graphs of the set-valued mapping $\zeta_{k+1} \mapsto$ $\mathcal{N}_{\left[-\lambda_{2} h^{2}, \lambda_{2} h^{2}\right]}\left(\zeta_{k+1}\right)$ and of the single-valued mapping $\zeta_{k+1} \mapsto-f\left(\zeta_{k+1}\right)$ have a unique intersection, see Fig. 2. Using the fact that both mappings in (11) are the inverse of their counterparts in (12), their intersection is also always unique.

This means that the controller in (10) is always uniquely defined from the available data at $t=t_{k}$. Let us recall that $b_{k}=-x_{1, k}-h \nu_{k}$. Fig. 2 is read as follows:

1) case $1, b_{k}<-h^{2} \lambda_{2}: \zeta_{k+1}=-h^{2} \lambda_{2}, \xi_{k+1}=1$, $\tilde{x}_{1, k+1}>0$,

2) case 2, $b_{k} \in\left[-h^{2} \lambda_{2}, h^{2} \lambda_{2}\right]: \zeta_{k+1}=b_{k} \in$ $\left[-h^{2} \lambda_{2}, h^{2} \lambda_{2}\right], \quad \xi_{k+1}=\frac{b_{k}}{-h^{2} \lambda_{2}} \in[-1,1]$, $\tilde{x}_{1, k+1}=0$,

3) case 3, $b_{k}>h^{2} \lambda_{2}: \zeta_{k+1}=h^{2} \lambda_{2}, \xi_{k+1}=-1$, $\tilde{x}_{1, k+1}<0$.

The value of $\tilde{x}_{1, k+1}$ can be computed easily in cases 1 and 3 by solving $g\left(\tilde{x}_{1, k+1}\right)= \pm \lambda_{2} h^{2}$. Using (10) we deduce the following algorithm for the calculation of the control input at time $t_{k}$ :

- data: $x_{1, k}$ and $\nu_{k}, a=h \lambda_{1}, h>0, \lambda_{1}>0$, $\lambda_{2}>0$.

- if $b_{k}<-h^{2} \lambda_{2}$, then $\sqrt{\left|\tilde{x}_{1, k+1}\right|}=$ $\frac{-a+\sqrt{a^{2}-4\left(b_{k}+\lambda_{2} h^{2}\right)}}{2} ; \quad \nu_{k+1}=\nu_{k}-h \lambda_{2}$; $u_{k}=-\lambda_{1} \sqrt{\left|\tilde{x}_{1, k+1}\right|}+\nu_{k+1}$, 


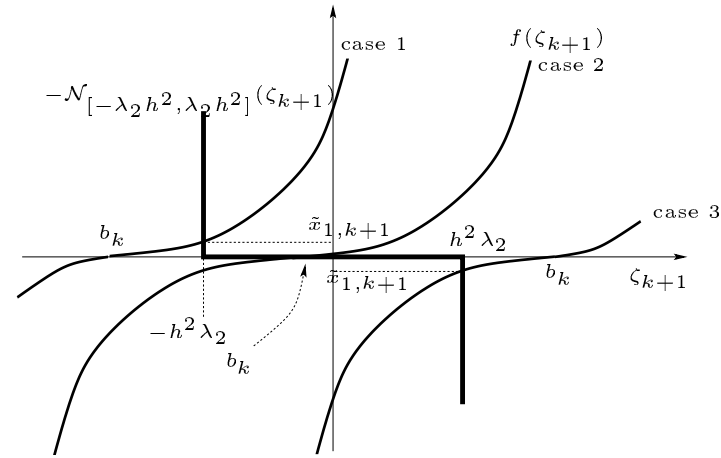

Fig. 2. Graphical interpretation of (12).

- else if $b_{k} \in\left[-h^{2} \lambda_{2}, h^{2} \lambda_{2}\right]$, then $u_{k}=\nu_{k+1}=-$ $\frac{x_{1, k}}{h}$,

- else if $b_{k}>-h^{2} \lambda_{2}$, then $\sqrt{\left|\tilde{x}_{1, k+1}\right|}=$ $\frac{-a+\sqrt{a^{2}+4\left(b_{k}-\lambda_{2} h^{2}\right)}}{2} ; \nu_{k+1}=\nu_{k}+h \lambda_{2} ; u_{k}=$ $\lambda_{1} \sqrt{\left|\tilde{x}_{1, k+1}\right|}+\nu_{k+1}$,

- terminate

\section{ANALYSIS OF THE DISCRETE-TIME}

\section{CLOSED-LOOP SYSTEM}

In the previous section, we have proved that the applied controller allows to generate a sequence of states $\tilde{x}_{1, k}$. Notice that in the absence of perturbation, we have $\tilde{x}_{1, k}=x_{1, k}$ for all $k \geq 0$ if $\tilde{x}_{1,0}=x_{1,0}$.

Definition 1. The discrete-time sliding surface is defined as $\Sigma_{d}=\left\{\left(\tilde{x}_{1, k}, \nu_{k}\right) \in \mathbb{R}^{2} \mid \tilde{x}_{1, k}=0, \nu_{k}=0\right\}$.

Let us characterize the fixed points of the unperturbed closed-loop system which is obtained from (8), setting $\varphi_{k}=0$ (hence $x_{1, k}=\tilde{x}_{1, k}$ for all $k$ ).

Lemma 3. Let $\varphi_{k}=0$ for all $k$. The unique fixed point of the unperturbed (or virtual) closed-loop system (8) is $\left(\tilde{x}_{1}^{\star}, \nu^{\star}\right)=(0,0)$.

Proof. Recall that in this case $\tilde{x}_{1, k}=x_{1, k}$. From (8) we obtain $0=-h \lambda_{1} \sqrt{\left|\tilde{x}_{1}^{\star}\right|} \operatorname{sgn}\left(\tilde{x}_{1}^{\star}\right)+h \nu^{\star}$ and $0 \in$ $-\lambda_{2} h \operatorname{sgn}\left(\tilde{x}_{1}^{\star}\right) \Leftrightarrow \tilde{x}_{1}^{\star}=0$. Thus $\nu^{\star}=0$.

Let us now analyse what happens on $\Sigma_{d}$, in the presence of a perturbation.

Lemma 4. Assume that the discrete-time state belongs to $\Sigma_{d}$ for all $t_{i}, i \leq k+1$. Then $x_{1, k}=h^{2} \lambda_{2} \xi_{k+1}^{1}$ for some $\xi_{k+1}^{1} \in[-1,1]$, while $x_{1, k+1}=h^{2} \lambda_{2} \xi_{k+1}^{2}+h \bar{\varphi}_{k}$ for some $\xi_{k+1}^{2} \in[-2,2]$. If $\tilde{x}_{1, k+2}=0$ and $\nu_{k+1}=0$ then $x_{1, k+1}$ will also satisfy $x_{1, k+1}=h^{2} \lambda_{2} \xi_{k+2}^{1}$ for some $\xi_{k+2}^{1} \in[-1,1]$
Proof. We have $\tilde{x}_{1, k+1}=0$ and $\nu_{k}=0$. From (9) it follows $0 \in x_{1, k}-h^{2} \lambda_{2} \operatorname{sgn}(0)$, which proves the first part for some selection $\xi_{k+1}^{1} \in[-1,1]$. The second part follows using (6): $x_{1, k+1} \in x_{1, k}-h^{2} \lambda_{2} \operatorname{sgn}(0)+h \bar{\varphi}_{k}$ so that $x_{1, k+1}=h^{2} \lambda_{2} \xi_{k+1}^{1}-h^{2} \lambda_{2} \xi_{k+1}^{3}+h \bar{\varphi}_{k}$ for some (possibly different from $\xi_{k+1}^{1}$ ) selection $\xi_{k+1}^{3} \in$ $[-1,1]$, and noting that $\xi_{k+1}^{1}-\xi_{k+1}^{3} \in[-2,2]$. The last statement is a corollary of the first one.

An important point is that Lemma 4 says nothing neither about the invariance of $\Sigma_{d}$, nor about its asymptotic or finite-time reachability: it just says that in case the system remains in $\Sigma_{d}$, then the closedloop system has an accuracy $h^{2}$ at $x_{1}$. The results in [8] are not applicable to the super-twisting algorithm, which does not belong to the class of systems analysed in that article. They also require the knowledge of a Lyapunov function for (1), whose level sets are convex, thus Lemma 1 is used. We now state the following result:

Lemma 5. Let $F: \mathbb{R}^{n} \rightrightarrows \mathbb{R}^{n}$ be an upper semicontinuous map such that $F(x)$ is nonempty convex and compact for any $x \in \mathbb{R}^{n}$. Let the differential inclusion

$$
\dot{x}(t) \in F(x(t))
$$

have the unique globally asymptotically stable equilibrium $x^{\star}=0$. If its strict Lyapunov function $V \in$ $C\left(\mathbb{R}^{n},[0,+\infty)\right) \cap C^{1}\left(\mathbb{R}^{n} \backslash\{0\},(0,+\infty)\right)$ has convex level sets, then any sequence $\left\{x_{k}\right\}_{i=0}^{+\infty}$ generated by the inclusion $0 \in x_{k+1}-x_{k}-h F\left(x_{k+1}\right)$ converges to zero as $k \rightarrow+\infty$, for any bounded initial data.

Proof. Let us denote the level set of $V(\cdot)$ as follows

$$
\Omega(\lambda)=\left\{x \in \mathbb{R}^{n} \mid V(x) \leq \lambda\right\},
$$

and define $\delta:(0,+\infty) \rightarrow(0,+\infty)$ as $\delta(\lambda)=$ $\inf _{x \in \partial \Omega(\lambda)} W(x)$, where $W(\cdot)$ is defined by (17), and $\partial \Omega(\lambda)$ corresponds to boundary of $\Omega(\lambda)$. Notice that the set $\Omega(\lambda)$ (as well as its boundary) is compact, due continuity and radial unboundedness of the Lyapunov function $V(\cdot)$. Moreover, the multivalued function $\Omega:[0,+\infty) \rightrightarrows \mathbb{R}^{n}\left(\right.$ resp. $\left.\partial \Omega:[0,+\infty) \rightrightarrows \mathbb{R}^{n}\right)$ is continuous in the Hausdorff metric. Hence, continuity/positivity of the function $\delta(\cdot)$ follows from continuity/positivity of the function $W(\cdot)$. From (17) we obtain:

$$
\sup _{x \in \partial \Omega(\lambda)} \sup _{\xi \in F(x)} \frac{\partial V}{\partial x} \xi \leq-\delta(\lambda)<0 \quad \text { if } \quad \lambda>0 .
$$

If $\xi \in F\left(x_{k+1}\right)$, then for any $x_{k}=x_{k+1}-h \xi$, one has

$$
\frac{\partial V\left(x_{k+1}\right)}{\partial x} \xi=\frac{\partial V\left(x_{k+1}\right)}{\partial x} \frac{x_{k+1}-x_{k}}{h} \leq-\delta\left(V\left(x_{k+1}\right)\right) .
$$


Since the level set $\Omega\left(V\left(x_{k+1}\right)\right)$ is convex, and $x_{k+1} \in$ $\partial \Omega\left(V\left(x_{k+1}\right)\right)$, then the last inequality immediately implies the point $x_{k}$ is separated from the convex compact set $\Omega\left(V\left(x_{k+1}\right)\right)$ by the tangential plane at the point $x_{k+1}$, as shown in Fig. 3 Hence, the distance

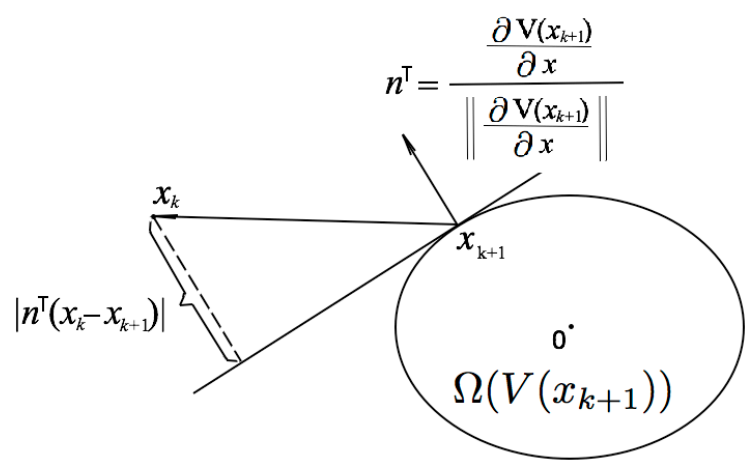

Fig. 3. Illustration of proof of Lemma. 5

from $x_{k}$ to $\Omega\left(V\left(x_{k+1}\right)\right)$ is greater than the distance from $x_{k}$ to the tangential plane (see Fig. 3). The corresponding distance $\left|n^{\top}\left(x_{k}-x_{k+1}\right)\right|$ can be estimated from below as follows:

$$
\begin{gathered}
\left|n^{\top}\left(x_{k}-x_{k+1}\right)\right|=\left|\frac{\frac{\partial V\left(x_{k+1}\right)}{\partial x}}{\left\|\frac{\left.\partial V x_{k+1}\right)}{\partial x}\right\|}\left(x_{k}-x_{k+1}\right)\right|= \\
=\frac{h\left|-\frac{\partial V\left(x_{k+1}\right)}{\partial x} \frac{\left(x_{k+1}-x_{k}\right)}{h}\right|}{\left\|\frac{\partial V\left(x_{k+1}\right)}{\partial x}\right\|} \geq d\left(V\left(x_{k+1}\right)\right),
\end{gathered}
$$

where

$$
d(\lambda)=h \delta(\lambda) \inf _{x \in \partial \Omega(\lambda)}\left\|\frac{\partial V(x)}{\partial x}\right\|^{-1}>0 \quad \text { if } \quad \lambda>0 .
$$

Since $\left\|\frac{\partial V(\cdot)}{\partial x}\right\|: \mathbb{R}^{n} \rightarrow \mathbb{R}$ is a continuous positive definite function then $d:(0,+\infty) \rightarrow(0,+\infty)$ is continuous as well. The latter means that

$$
V\left(x_{k+1}\right)-V\left(x_{k}\right)<-\varepsilon_{k},
$$

where $\varepsilon_{k}>0$ is separated from zero while $V\left(x_{k+1}\right)$ is separated from zero. Therefore, we derive that the sequence $V\left(x_{k}\right)$ is monotone decreasing to zero as $k \rightarrow+\infty$.

Corollary 1. Suppose that the perturbation $\Delta(t) \equiv 0$, $\lambda_{1}>0$ and $0<\lambda_{2}<\frac{\lambda_{1}^{2}}{2 \sqrt{2}}$. The origin of the discretetime closed-loop system (8), is globally asymptotically stable in the sense of Lyapunov.

Proof. Recall that in such a case $\tilde{x}_{1, k}=x_{1, k}$, provided the initial values coincide. Thus, (8) is the discretization of (1) since also $x_{2}=\nu$. Taking into account
Lemma 1, we conclude from Lemma 5 that the state of the system (8), which rewrites

$\left\{\begin{array}{l}x_{1, k+1}=x_{1, k}-h \lambda_{1} \sqrt{\left|x_{1, k+1}\right|} \operatorname{sgn}\left(x_{1, k+1}\right)+h \nu_{k+1} \\ \nu_{k+1} \in \nu_{k}-\lambda_{2} h \operatorname{sgn}\left(x_{1, k+1}\right),\end{array}\right.$,

converges asymptotically to zero, i.e., the implicit scheme provides a globally asymptotically stable discretization.

Remark 2. We have not yet proved that $\tilde{x}_{1, k}$ attains the origin in a finite number of steps, even in the unperturbed case. For the moment only asymptotic convergence holds. In [2], [12] it has been possible to prove that the state $\tilde{x}_{1, k}$ of the unperturbed virtual system, converges to zero in a finite number of steps, even in the presence of a disturbance. This was done either by direct calculations, or from a suitable Lyapunov function analysis. In our case, the closed-loop system with perturbation is given by:

$$
\begin{aligned}
& x_{1, k+1}=x_{1, k}-h \lambda_{1} \sqrt{\left|\tilde{x}_{1, k+1}\right|} \xi_{k+1}+h \nu_{k+1}+h \bar{\varphi}_{k} \\
& \varphi_{k+1}=\varphi_{k}+h \bar{\Delta}_{k} \\
& \nu_{k+1}=\nu_{k}-\lambda_{2} h \xi_{k+1} \\
& 0 \in f\left(-\lambda_{2} h^{2} \xi_{k+1}\right)-\mathcal{N}_{[-1,1]}\left(\xi_{k+1}\right) \\
& 0 \in g\left(\tilde{x}_{1, k+1}\right)+\lambda_{2} h^{2} \operatorname{sgn}\left(\tilde{x}_{1, k+1}\right) \\
& g\left(\tilde{x}_{1, k+1}\right)=\tilde{x}_{1, k+1}+h \lambda_{1} \sqrt{\left|\tilde{x}_{1, k+1}\right|} \operatorname{sgn}\left(\tilde{x}_{1, k+1}\right) \\
& \quad-x_{1, k}-h \nu_{k} .
\end{aligned}
$$

It is not clear how to prove that $\tilde{x}_{1, k}$ reaches zero in a finite number of steps when $\bar{\varphi}_{k} \neq 0$ for all $k \geq 0$. The so-called equivalent controller is obtained from (16) by setting $x_{1, k+1}=x_{1, k}=0$ and $\nu_{k+1}=\nu_{k}$. It gives $u_{k}^{e q}=-\varphi_{k}$, which means that the equivalent controller compensates for the disturbance with a one-step delay. However it is not implementable since $\varphi_{k}$ is unknown. Nevertheless we can now state the following:

Corollary 2. Assume that $\Delta(t) \equiv 0$. The sliding surface $\Sigma_{d}$ is attained in a finite number of steps and is invariant.

Proof. We know from Corollary 1 that for any $\epsilon>0$, there exists $0<n<+\infty$ such that $\left|x_{1, k}\right|<\epsilon$ and $\left|\nu_{k}\right|<\epsilon$ for all $k \geq n$. Choosing $n$ large enough (but finite for $h>0$ ) it follows that $-x_{1, k}-$ $h \nu_{k} \in\left[-h^{2} \lambda_{2}, h^{2} \lambda_{2}\right]$ for all $k \geq n$. As shown above, in such a case (named case 2), one has $u_{k}=$ $-\frac{x_{1, k}}{h}$. We can now use (7) (which is equivalent to (6) since the disturbance is null) to deduce that $x_{1, k+1}=x_{1, k}+h u_{k}=0$. From (8), we infer that $\nu_{k+1}=\nu_{k}-\lambda_{2} h \frac{-x_{1, k}-h \nu_{k}}{-h^{2} \lambda_{2}}=-\frac{x_{1, k}}{h}$. Therefore, 
$-x_{1, k+1}-h \nu_{k+1}=-h \nu_{k+1}=x_{1, k}$ and taking $n$ large enough (or equivalently $\epsilon$ small enough) we deduce that $-x_{1, k+1}-h \nu_{k+1} \in\left[-h^{2} \lambda_{2}, h^{2} \lambda_{2}\right]$. Consequently, $u_{k+1}=-\frac{x_{1, k+1}}{h}=0$, and $\nu_{k+2}=\nu_{k+1}-h \lambda_{2} \xi_{k+2}=$ $\nu_{k+1}-h \lambda_{2} \frac{-x_{1, k+1}-h \nu_{k+1}}{-h^{2} \lambda_{2}}=0$. Repeating the same argument, it follows that $x_{1, k}=\nu_{k+1}=0$ for all $k \geq n$.

It is noteworthy that in the absence of perturbation, the discrete-time system not only attains $\Sigma_{d}$ in a finite number of steps, but then stays on it with zero input and zero oscillations (suppression of the digital chattering). This is coherent with the results in [1], [2], [12], [14].

\section{Simulations}

For simulation the plants is discretized as in (6) using the explicit Euler method, and the explicit Euler discretization of the controller reads as $u_{k}=$ $-\lambda_{1} \sqrt{\left|x_{1, k}\right|} \operatorname{sgn}\left(x_{1, k}\right)+\nu_{k}$, and $\nu_{k+1}=\nu_{k}-$ $\lambda_{2} h \operatorname{sgn}\left(x_{1, k}\right)$. Let us select the following parameters: $\lambda_{1}=10, \lambda_{2}=6$, which satisfy all needed restrictions, and initial conditions $x_{1}(0)=\nu(0)=10$, then let us compare the explicit discretization Euler method, and the implicit one proposed in this note. First, let $\Delta(t)=\varphi(0)=0$, then the behavior of a homogeneous norm $e_{k}=\left|x_{1, k}\right|+\nu_{k}^{2}$ for both methods (solid red line corresponds to the explicit Euler method, and dashed blue line represents the implicit one) is shown in Fig. 4 in a logarithmic scale for two values of constant discretization step ( $h=0.1 \mathrm{~s}$ and $h=0.001 \mathrm{~s}$ ). The corresponding values of the norm of control $\left|u_{k}\right|$ are presented in Fig. 5, where we can notice that the control in the implicit case is converging exactly to zero in a finite time, while that obtained by the explicit discretization, has a constant amplitude after a transient. The plot for $\Delta(t)=2\left(1+\sin \left(2 t^{2}\right)\right)$, $h=0.001 \mathrm{~s}$ and $\varphi(0)=0$ are given in Fig. 6, and the corresponding control amplitudes of both methods, can be compared in Fig. 7: again the control generated by the implicit method has smaller amplitude, and much less chattering. The numerical results confirm the theoretical findings, and a much better accuracy for the proposed implicit scheme (though we have no proof for the latter property). See also [7, Theorems $1,2]$ for the analysis and simulation of the explicit method for HOSM controllers.

\section{CONCLUSIONS}

In this article the properties of the time-discretization of the super-twisting algorithm are analysed. An implicit Euler scheme is used to define the discretetime system. First it is shown that the continuous-time
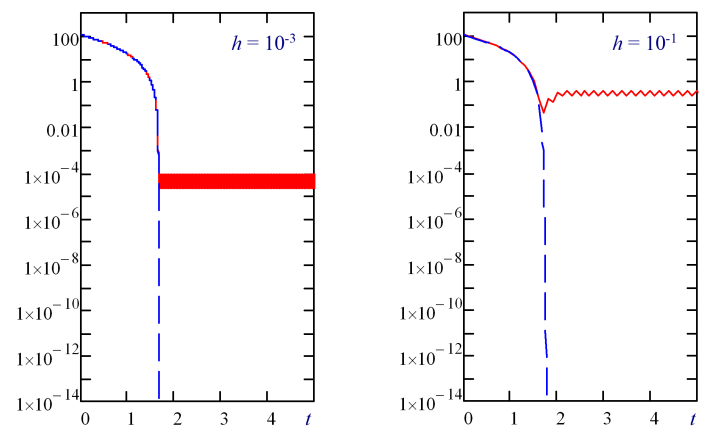

Fig. 4. Regulation error $e_{k}$ without perturbation for $h=0.001 \mathrm{~s}$ (left) and $h=0.1 \mathrm{~s}$ (right).
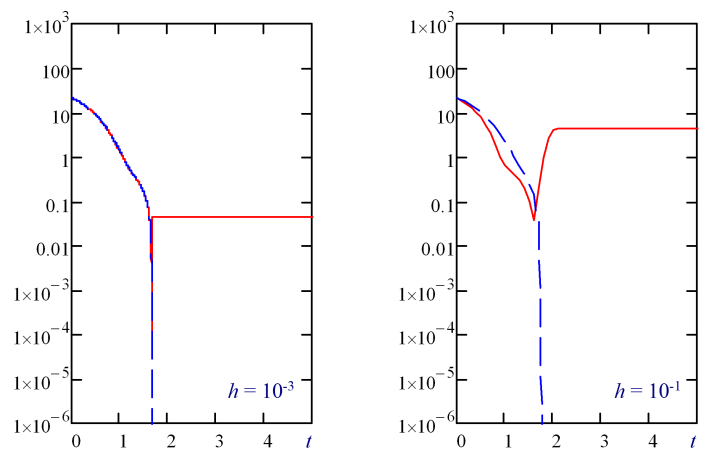

Fig. 5. Control amplitudes in the case without perturbation for $h=$ $0.001 \mathrm{~s}$ (left) and $h=0.1 \mathrm{~s}$ (right).

super-twisting controller, yields a closed-loop system which admits a strict Lyapunov function whose level sets are convex. Then it is proved that the discrete-time controller can be uniquely computed from available data, as the solution of two generalized equations. Finally it is shown that the existence of a Lyapunov function for the continuous-time closed-loop system, with convex level sets, implies the global asymptotic stablity of the discrete-time closed-loop system. Convergence

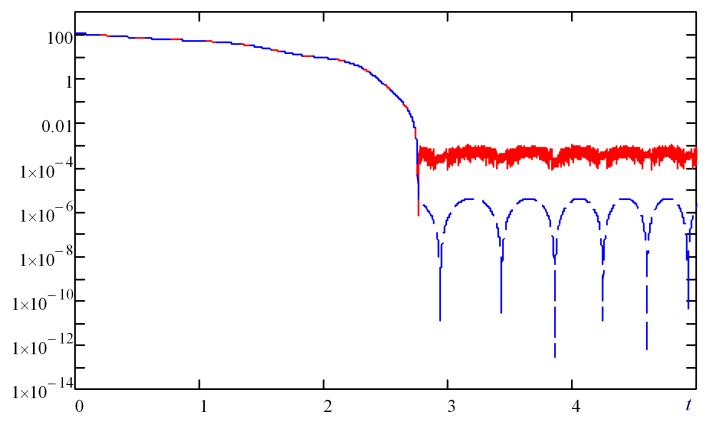

Fig. 6. Regulation error $e_{k}$ with perturbation for $h=0.001 \mathrm{~s}$; explicit (solid red line) and implicit Euler method (dashed blue line) 


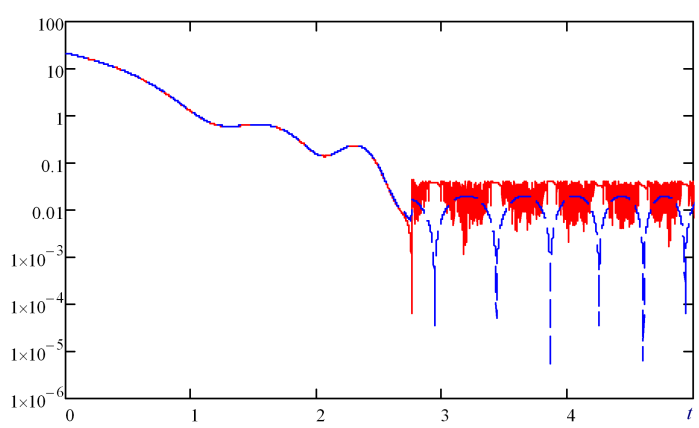

Fig. 7. Control amplitudes with perturbation for $h=0.001$ s; explicit (solid red line) and implicit Euler method (dashed blue line)

to the origin in a finite number of steps is proved in the unperturbed case. Numerical simulations confirm that the implicit discretization, supersedes the explicit one. Future investigations should focus on robustness and accuracy properties, and extensions towards HOSMC.

\section{A. Strict Lyapunov functions}

Let us recall that a function $V \in C\left(\mathbb{R}^{n},[0,+\infty)\right) \cap$ $C^{1}\left(\mathbb{R}^{n} \backslash\{0\},(0,+\infty)\right)$ is said to be a strict Lyapunov function to the system (14) if $V(0)=0$, $V(x) \rightarrow+\infty$ as $\|x\| \rightarrow+\infty$ and there exists $W \in C\left(\mathbb{R}^{n} \backslash\{0\},(0,+\infty)\right)$ such that

$$
\sup _{\xi \in F(x)} \frac{\partial V}{\partial x} \xi \leq-W(x) \quad \forall x \in \mathbb{R}^{n} \backslash\{0\} .
$$

Such a strict Lyapunov function always exists for globally asymptotically stable systems (14), see [5].

\section{REFERENCES}

[1] V. Acary and B. Brogliato. Implicit Euler numerical scheme and chattering-free implementation of sliding mode systems. Systems and Control Letters, 59:284-293, 2010.

[2] V. Acary, B. Brogliato, and Y. Orlov. Chattering-free digital sliding-mode control with state observer and disturbance rejection. IEEE Transactions on Automatic Control, 57(5):10871101, 2012.

[3] M.T.S. Aung, Z. Shi, and R. Kikuuwe. A new parabolic sliding mode filter augmented by a linear low-pass filter and its application to position control. ASME J. of Dynamic Systems, Measurement and Control, 140:041005, 2018.

[4] B. Brogliato and A. Polyakov. Globally stable implicit Euler time-discretization of a nonlinear single-input sliding-mode control system. In Proc. IEEE 54th Int. Conf. on Decision and Control, pages 5426-5431, Osaka, Japan, December 2015.

[5] F. H. Clarke, Yu. S. Ledyaev, and R.J. Stern. Asymptotic stability and smooth Lyapunov functions. Journal of Differential Equations, 149:69-114, 1998.

[6] L. Derafa, A. Benallegue, and L. Fridman. Super twisting control algorithm for the attitude tracking of a four rotors UAV. Journal of the Franklin Institute, 349(2):685-699, 2012.

[7] H. Du, J. Zhai, M.Z.Q. Chen, and W. Zhu. Robustness analysis of a continuous higher order finite-time control system under sampled-data control. IEEE Transactions on Automatic Control, 64(6):2488-2494, 2019.
[8] D. Efimov, A. Polyakov, A. Levant, and W. Perruquetti. Realization and discretization of asymptotically stable homogeneous systems. IEEE Transactions on Automatic Control, 62(11):5962-5969, 2017.

[9] F. Facchinei and J.S. Pang. Finite-Dimensional Variational Inequalities and Complementarity Problems, volume I of $\mathrm{Op}$ erations Research. Springer-Verlag, New York, 2003.

[10] L. Fridman. Sliding Mode Enforcement after 1990: Main Results and Some Open Problems, volume 412 of LNCIS, pages 3-57. Springer - Verlag, Berlin Heidelberg, 2011.

[11] Z. Galias and X. Yu. Euler's discretization of single input sliding-mode control systems. IEEE Transactions on Automatic Control, 52(9):1726-1730, September 2007.

[12] O. Huber, V. Acary, and B. Brogliato. Lyapunov stability and performance analysis of the implicit discrete sliding mode control. IEEE Transactions on Automatic Control, 61(10):30163030, 2016.

[13] O. Huber, V. Acary, B. Brogliato, and F. Plestan. Implicit discrete-time twisting controller without numerical chattering: Analysis and experimental results. Control Engineering Practice, 46(1):129-141, 2016.

[14] O. Huber, B. Brogliato, V. Acary, A. Boubakir, F. Plestan, and B. Wang. Experimental results on implicit and explicit timediscretization of equivalent-control-based sliding-mode control. In L. Fridman, J.P. Barbot, and F. Plestan, editors, Recent Trends in Sliding-Mode Control, number 102 in Control, Robotics and Sensors, pages 207-236. IET, The Institution of Engineering and Technology, 2016.

[15] R. Kikuuwe, H. Fujimoto S. Yasukouchi, and M. Yamamoto. Proxy-based sliding mode control: A safer extension of PID position control. IEEE Transactions on Robotics, 26(4):670 683,2010

[16] S. Koch and M. Reichhartinger. Discrete-time equivalents of the super twisting algorithm. Automatica, 107:190-199, 2019.

[17] A. Levant. On fixed and finite time stability in sliding mode control. In 52nd IEEE Conference on Decision and Control, pages 4260-4265, Firenze, Italy, 2013.

[18] F. Miranda-Villatoro, B. Brogliato, and F. Castanos. Multivalued robust tracking control of Lagrange systems: continuous and discrete-time algorithms. IEEE Transactions on Automatic Control, 62(9):4436-4450, 2017.

[19] F. Miranda-Villatoro, B. Brogliato, and F. Castanos. Set-valued sliding-mode control of uncertain linear systems: continuous and discrete-time analysis. SIAM J. on Control and Optimization, 56(3):1756-1793, 2018.

[20] J. Moreno and M. Osorio. Strict Lyapunov functions for the super-twisting algorithm. IEEE Transactions on Automatic Control, 57(4):1035 - 1040, 2012.

[21] Y. Orlov. Finite time stability and robust control synthesis of uncertain switched systems. SIAM Journal of Control and Optimization, 43(4):1253-1271, 2005.

[22] A. Polyakov, D. Efimov, and W. Perruquetti. Finite-time and Fixed-time Stabilization: Implicit Lyapunov Function Approach. Automatica, 51(1):332-340, 2015.

[23] A. Polyakov and A. Poznyak. Reaching time estimation for "super-twisting" second order sliding mode controller via Lyapunov function designing. IEEE Transactions on Automatic Control, 54(8):1951-1955, 2009.

[24] R. Seeber and M. Horn. Stability proof for a well-established super-twisting parameter setting. Automatica, 84:241-243, 2017.

[25] B. Wang, B. Brogliato, V. Acary, A. Boubakir, and F. Plestan. Experimental comparisons between implicit and explicit implementations of discrete-time sliding mode controllers: Toward input and output chattering suppression. Control Systems Technology, IEEE Transactions on, 23(5):2071-2075, Sept 2015. 